\title{
Evidence for a Role of the Drosophila melanogaster suppressor of sable Gene in the Pre-mRNA Splicing Pathway
}

\author{
ROBERT A. FRIDELL† AND LILLIE L. SEARLES* \\ Department of Biology, University of North Carolina at Chapel Hill, \\ Chapel Hill, North Carolina 27599-3280
}

Received 30 July 1993/Returned for modification 26 September 1993/Accepted 6 October 1993

\begin{abstract}
Recessive mutations of the Drosophila melanogaster suppressor of sable [su(s)] gene result in elevated accumulation of RNA from vermilion (v) mutant alleles that have an insertion of the 7.5-kb retrotransposon 412 in the first exon of the $v$ gene. During transcription of such a $v$ mutant gene, the 412 sequences are incorporated into the primary transcripts and are subsequently removed by splicing at cryptic sites within 412 sequences. In a $s u(s)^{+}$background, the level of these unusually spliced transcripts is exceedingly low, and $s u(s)$ mutations increase their accumulation. We previously proposed that $v$ RNA levels are elevated in $s u(s)$ mutants because of increased recognition of the cryptic splice sites, and the aim of this study was to test this hypothesis. We generated a $v$ mutant derivative with a smaller 412 insertion, introduced alterations into the 412 -associated splice sites, and examined the effect of $s u(s)$ mutations on expression of these derivatives after germ line transformation. To increase overall expression levels, the $v$ promoter was replaced with the stronger Metallothionein (Mtn) gene promoter. We found that transformants bearing a $v$ derivative with 480 bp of 412 sequences accumulate both transcripts, with 412 sequences spliced out and transcripts that retain 412 sequences. Mutations of $s u(s)$ increase the levels of both transcript classes without affecting the relative amounts of the two forms. Strikingly, replacement of the cryptic 5' splice sites with a 5' consensus produces the same effect as, and eliminates the response to, a $s u(s)$ mutation. In addition, we demonstrated that mutations of $s u(s)$ lead to increased accumulation of $v$ transcripts even when the previously identified cryptic $4125^{\prime}$ and $3^{\prime}$ splice sites were destroyed and that other cryptic splice sites reside within Mtn and 412 sequences. These results indicate that the $v$ mutant transcripts are stabilized by assembly of the 412 sequences into splicing complexes and support the hypothesis that splicing complexes more readily assemble on cryptic splice sites in $s u(s)$ mutants.
\end{abstract}

Transposition of mobile DNA elements is a common cause of mutations in eukaryotes. In Zea mays and Drosophila melanogaster, a few cases have been described wherein a transposon has inserted into an exon of a particular gene, and after being incorporated into the primary RNA transcript, the majority of the transposon sequences are removed from the pre-mRNA by splicing (22). Thus, low levels of nearly wild-type-size transcripts are generated, despite the presence of a transposon insertion within transcribed sequences. In $D$. melanogaster, splicing events of this type have been described for certain mutant alleles of vermilion (v) and yellow $(y)$, which are associated with retrotransposon 412 and P-element insertions, respectively $(3,6)$. Both the $v$ and $y$ mutations are suppressed by recessive mutations at the unlinked suppressor of sable $[s u(s)]$ gene. We are using the $v$ gene as a model to investigate the mechanism of suppression.

The $v$ gene encodes an enzyme, tryptophan oxygenase, which is required for brown eye pigment synthesis $(20,31)$. The alleles $v^{1}, v^{2}$, and $v^{k}$ (collectively referred to as $v^{s}$ ) are phenotypically suppressed by $s u(s)$ mutations. Each $v^{s}$ allele has an identical insertion of a 7.5-kb 412 retrotransposon in the first $v$ exon, 36 nucleotides (nt) downstream of the major transcription start site and 21 nt upstream of the translation start codon $(24,25)$. The 412 element is oriented such that

\footnotetext{
* Corresponding author. Phone: (919) 966-4989. Fax: (919) 9621625.

$\dagger$ Present address: Howard Hughes Medical Institute, Duke University Medical Center, Durham, NC 27710.
}

normal transcription of the element would proceed in the opposite direction as transcription of the $v$ gene (24). Although the insertion is in an exon, trace amounts of apparently wild-type-size $v$ transcripts accumulate in $v^{s}$ mutants, and mutations at the $s u(s)$ gene result in an approximately fivefold increase in the accumulation of these transcripts (21, 24). We have previously shown that the nearly wild-typesize $v^{s}$ transcripts are generated by splicing of the majority of the 412 retrotransposon sequences from the $v^{s}$ pre-mRNA at cryptic splice sites located near the ends of the transposon (3). Four 5' splice donor sites were found to be alternatively spliced to a single $3^{\prime}$ splice acceptor. These splice sites are located within the long terminal repeat (LTR) sequences of the 412 retrotransposon and are derived from the normally nontranscribed strand of the 412 element. They are, therefore, not sites that are normally used to process 412 transcripts. Comparison of the cryptic 412 splice sites to actual splice sites of several $D$. melanogaster genes revealed that the $4125^{\prime}$ splice sites are atypically poor matches to the invertebrate consensus splice site sequence (3).

The $s u(s)$ gene encodes a nuclear protein with sequence features that suggest a role in RNA processing (29). Previously, we proposed that the $s u(s)$ protein may function to prevent splicing complexes from forming on poor $5^{\prime}$ splice sites; thus, the 412 element would be more readily recognized as an intron in $s u(s)$ mutants. Here we have constructed derivatives of a $v^{s}$ allele with a smaller 412 insertion and with alterations in the 412-associated splice sites. The expression of these derivatives was examined in $s u(s)$ wildtype and $s u(s)$ mutant backgrounds after germ line transfor- 
mation. The results presented in this report provide strong support for a role of the $s u(s)$ protein in the pre-mRNA splicing pathway.

\section{MATERIALS AND METHODS}

Plasmid constructions. The $\mathrm{v}^{\mathrm{k}} \mathrm{LTR}, \mathrm{Mtnv}^{\mathrm{k}} \mathrm{LTR}, \mathrm{Mtnv}^{\mathrm{k}}$ LTR5' con, Mtnv ${ }^{k} L T R \Delta 5^{\prime} S S$, and Mtnv ${ }^{k} L T R \Delta 5^{\prime}, 3^{\prime}$ SS constructions were made with the recombinant PCR technique described by Higuchi (8). Desired mutations were confirmed by dideoxynucleotide sequencing. The $\mathbf{v}^{\mathbf{k}} \mathrm{LTR}$ construction includes approximately $3.2 \mathrm{~kb}$ of $v^{+} 5^{\prime}$ flanking sequences and $300 \mathrm{bp}$ of $v^{+} 3^{\prime}$ flanking sequences and has a single 412 LTR at the insertion site of the 412 element in the $v^{s}$ alleles. In the Metallothionein (Mtn): $v$ chimeric constructions, the $v^{+} 5^{\prime}$ HindIII-XhoI fragment $(-3200$ to +28$)$ was replaced with an EcoRI-SalI fragment isolated from plasmid pMCR11 (obtained from G. Maroni) which contains the Mtn core promoter and several copies of a metal response element inserted into the EcoRI and SmaI sites of the pUC18 cloning vector. The Mtn EcoRI-SalI fragment contains Mtn sequences from -373 to +54 and approximately $15 \mathrm{bp}$ of pUC18 polylinker sequences. For germ line transformation, all of the constructions were introduced into unique NotI and SalI sites of the $D$. melanogaster transformation vector pDM30 (17).

D. melanogaster strains and germ line transformation. Flies were reared at $25^{\circ} \mathrm{C}$ on standard food. The mutant $D$. melanogaster alleles used in this study have been described elsewhere (12). A $v^{36 f}$ rosy ${ }^{506}$ strain was used as a host strain for germ line transformation. The $v^{36 f}$ allele has an insertion of a B104/roo retrotransposon in the fourth $v$ intron and produces $v$ transcripts of very low abundance that terminate within the transposon insertion (24). Germ line transformation was carried out as previously described (9). Crosses with standard balancer chromosomes were used to generate homozygous transgenic lines in $s u(s)^{+}$and $s u(s)^{51 c 15}$ backgrounds. The $s u(s)^{51 c 15}$ mutant has a gypsy element insertion in the first intron of the $s u(s)$ gene (30) and produces a reduced level of apparently normal protein (17a). Transgene insertions were verified by Southern analysis. For this analysis, genomic DNA was isolated from 1- to 5-day-old adult flies (1) and fractionated on $0.8 \%$ agarose gels. Transfer to nylon membranes, prehybridization, hybridization, and posthybridization washes were performed as described by Southern (27). ${ }^{32} \mathrm{P}$-labeled DNA probes were prepared by random priming (2).

RNA isolation and Northern (RNA) blot analysis. Total RNA was isolated from 1- to 5-day-old adult flies as described by Fridell and Searles (4). Poly (A) ${ }^{+}$RNA was prepared with a PolyATtract mRNA isolation kit (Promega). For Northern blot analysis, 2 to $5 \mu \mathrm{g}$ of poly $(\mathrm{A})^{+}$RNA was fractionated on $1.2 \%$ agarose-formaldehyde gels (13) and blotted onto nylon membranes (28). Prehybridization, hybridization to riboprobes (see below), and posthybridization washes were performed as described by Zinn et al. (32). Northern blots were simultaneously probed with $v$ and internal control riboprobes (see below). High-specific-activity $v$ riboprobes were prepared as described by Melton et al. (16) except that $200 \mu \mathrm{Ci}$ of [ $\left.{ }^{32} \mathrm{P}\right] \mathrm{CTP}(800 \mathrm{Ci} / \mathrm{mmol})$ was used in each reaction without the addition of cold CTP. To avoid an interfering signal from the injection host strain, the $v$ riboprobe was derived from $v$ sequences downstream of the $B 104$ insertion in the $v^{36 f}$ allele (see above). The internal control riboprobes, $r p 49(18)$ and $M t n(14)$, were prepared as described by Melton et al. (16). Prehybridization, hybridiza- tion to the DNA probes, and posthybridization washes were performed as described by O'Hare et al. (19).

Analysis of partial cDNA clones of the Mtnvk LTR constructions. PCR-amplified cDNA clones of the Mtnvk LTR, Mtnv $v^{k} L T R 5^{\prime}$ con, and $M t n v^{k} L T R \Delta 5^{\prime}, 3^{\prime} S S$ constructions were obtained and sequenced as previously described (3) except that $20 \mu \mathrm{g}$ of total adult RNA was used as a template for first-strand cDNA synthesis. Oligonucleotide 5276, which is complementary to the $M t n$ sense strand ( +2 to +20 [14]) and has an XhoI site added to its 5 ' end, and oligonucleotide 2052 (3), which spans the EcoRI site in exon 4 of the $v$ gene, were used as primers for the PCR. Amplified cDNA fragments were cloned into the plasmid vector pGEM7Zf (Promega) through the use of the XhoI site contained within the upstream Mtn primer and an internal ClaI site located in $v$ exon 3 (Fig. 1). The splicing patterns of the majority of the cDNA clones were determined by dideoxynucleotide sequencing. However, the splicing patterns of some of the Mtnvk ${ }^{k}$ TR and Mtnv ${ }^{k}$ LTR5'con cDNA clones were deduced on the basis of characteristic sizes of the cloned cDNA fragments. For the Mtnv ${ }^{k} L T R \Delta 5^{\prime} S S$ construction, PCR-amplified CDNA products were isolated from agarose gels and used to generate single-stranded DNA as described by Kreitman and Landweber (11). The single-stranded DNA served as a template for dideoxynucleotide sequencing.

\section{RESULTS}

Analysis of a $v$ construction with a single 412 LTR insertion. On the basis of the unusual splicing of the 412 retrotransposon sequences from the $v^{s}$ primary transcripts, we previously proposed that $s u(s)$ mutations may result in the stabilization of splicing complexes at the cryptic 412 splice sites and thereby facilitate splicing of the 412 intron (3). However, it is also possible that $s u(s)$ mutations can increase the frequency of transcription initiation of the $v^{s}$ alleles or increase the stability of the $v^{s}$ transcripts. To test these possibilities, we decided to make changes in the cryptic 412 splice sites of a $v^{s}$ allele, introduce these altered alleles into $D$. melanogaster by transformation, and analyze their expression in $s u(s)$ wild-type and mutant backgrounds. As a first step in this approach, we constructed a smaller derivative of a $v^{s}$ allele that would be amenable to in vitro manipulation. Splicing of 412 sequences from the $v^{s}$ premRNA occurs from $5^{\prime}$ splice sites located near the end of one of the LTRs of the 412 retrotransposon to a $3^{\prime}$ splice site located near the opposite end of the other LTR (Fig. 1A). However, since the LTRs are direct repeats, both the $5^{\prime}$ and $3^{\prime}$ splice site sequences are present in both LTRs, and we expected that splicing could occur between splice sites located within the same LTR. Therefore, we made a construction which included the entire $v$ transcription unit, 3,200 bp of $v^{+} 5^{\prime}$ flanking sequences, $300 \mathrm{bp}$ of $v^{+} 3^{\prime}$ flanking sequences, and a 480-bp 412 LTR at the insertion site of the 7.5-kb 412 retrotransposon in the $v^{s}$ alleles. This construction, $v^{k} L T R$, was introduced into $D$. melanogaster by P-element-mediated germ line transformation. Analysis of PCRamplified cDNA clones from this construction revealed that the 412 LTR sequences were in fact spliced from the pre-mRNA at the same $5^{\prime}$ and $3^{\prime}$ splice sites previously identified in splicing the intact 412 element from the $v^{s}$ pre-mRNA. However, the level of expression of this construction was low, and by Northern blot analysis it was difficult to assess the effect of $s u(s)$ mutations on the accumulation of these transcripts. In an effort to circumvent the low level of expression and to test whether the $v$ promoter is 
A.
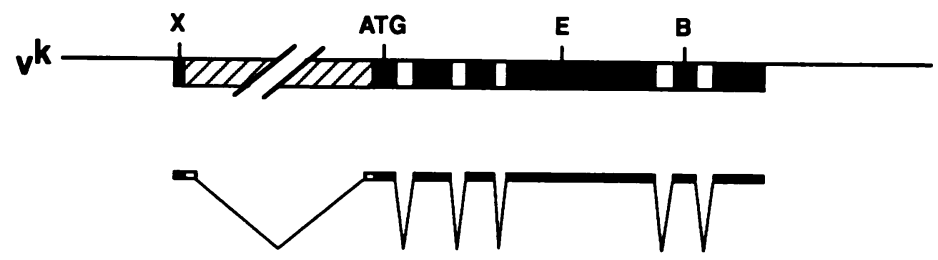

B.

B.

Mtnv $\mathrm{k}_{\mathrm{LTR}}$

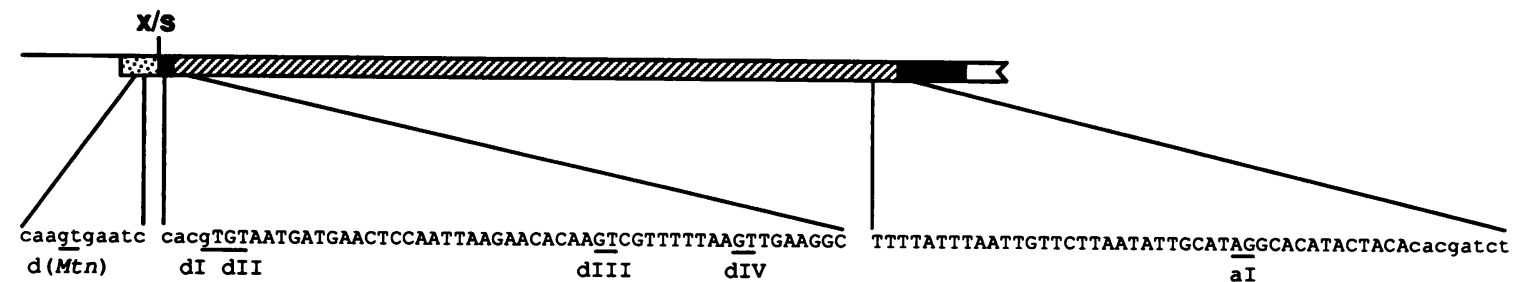

Mtnv' ${ }^{\mathrm{k}}$ LTR5 'con

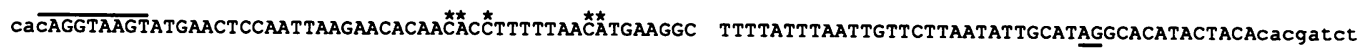

Mtnv ${ }^{k}$ LTR $\Delta 5$ 'SS

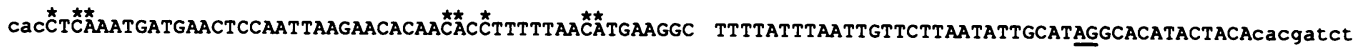

Mtnv ${ }^{\mathrm{L} T R \Delta 5^{\prime}, 3^{\prime} \mathrm{SS}}$

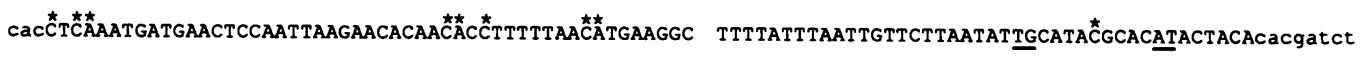

FIG. 1. Splicing pattern of the $v^{k}$ transcripts and alterations of the cryptic 412 splice sites. (A) Molecular map of the $v^{s}$ alleles. The inserted 412 retrotransposon is represented by the hatched box, $v$ exons are represented by shaded boxes, and $v$ introns are represented by open boxes. The ATG indicates the start of the $v$ open reading frame. The splicing pattern of the $v^{k}$ transcripts is schematically diagrammed immediately beneath the molecular map. (B) Constructions used in this study. In construction Mtnv $v^{k}$ TR, the $v$ promoter was replaced by the stronger Mtn promoter (stippled box) and the $7.5-\mathrm{kb} 412$ retrotransposon was replaced by a single 480-bp 412 LTR (hatched rectangle). The sequences in the vicinity of the previously described cryptic 412 splice sites (dI, dII, dIII, dIV, and aI) are shown, with the highly conserved GT and AG dinucleotides of the splice donor and acceptor sites underlined. The sequence of the cryptic donor site in the Mtn leader [d(Mtn); see text] is also shown. Sequences which are derived from the 412 transposon are in uppercase letters, whereas those from the $v$ or Mtn genes are in lowercase letters. In the Mtnv ${ }^{\mathrm{k}} \mathrm{LTR} 5$ 'con construction, the region including dI and dII has been converted to a consensus $5^{\prime}$ splice site, which is denoted by the line above the sequence. Additional changes to the cryptic splice sites in this and the Mtnv ${ }^{k}$ LTR $\Delta 5^{\prime} S S$ and Mtnv' ${ }^{k}$ TR $\Delta 5^{\prime}, 3^{\prime} S S$ constructions are indicated with asterisks. In addition, the positions of the two non-AG $3^{\prime}$ splice sites (see text) of Mtnv'LTR $\Delta 5^{\prime}, 3^{\prime}$ SS are underlined. Restriction enzyme abbreviations: B, BamHI; E, EcoRI; S, SalI; X, XhoI.

required for the effect of $s u(s)$ mutations, the $v 5^{\prime}$ fragment $(-3200$ to +25$)$ of the $v^{k}$ LTR construction was replaced with a fragment containing the promoter of the $D$. melanogaster Mtn gene and $54 \mathrm{bp}$ of Mtn leader sequences (14). This construction was designated Mtnv ${ }^{k}$ LTR (Fig. 1B). Poly(A) ${ }^{+}$ RNA isolated from transgenic flies bearing homozygous copies of Mtnvk $v^{k}$ TR in both $s u(s)^{+}$and $s u(s)$ mutant backgrounds was examined by Northern blot analysis (Fig. 2). Two $v$-specific transcripts of 1.4 and $1.9 \mathrm{~kb}$ were detected (Fig. 2, lanes 3 to 6). These bands correspond in size to transcripts which retain the 412 LTR sequences but have the $v$ introns spliced out $(1.9 \mathrm{~kb})$ and fully spliced transcripts which have lost the 412 LTR sequences as well as $v$ introns $(1.4 \mathrm{~kb})$. To verify the identity of the $1.9-\mathrm{kb}$ transcript, the Northern blot shown in Fig. 2 was stripped and reprobed with 412 LTR sequences. Only the $1.9-\mathrm{kb}$ band hybridized to the 412 LTR sequences, consistent with its representing transcripts which retain the 412 LTR (data not shown). Mutations at the $s u(s)$ gene increase the accumulation of both the fully spliced 1.4-kb transcripts and the 1.9-kb LTR-containing transcripts to the same extent (Fig. 2; compare lane 3 with lane 4 and lane 5 with lane 6 ). This result indicates that neither the $v$ promoter nor the majority of the 412 retrotransposon sequences are necessary for the effect of $s u(s)$ mutations. Also, as judged by the similar increases in the levels of the 1.4- and 1.9-kb transcripts, $s u(s)$ mutations

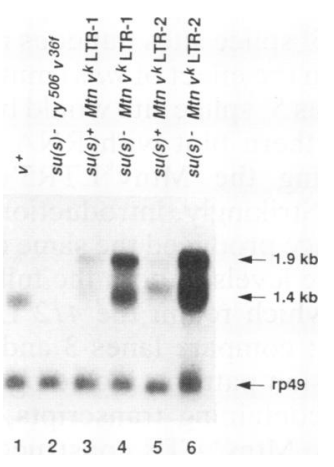

FIG. 2. Northern blot analysis of RNA isolated from transgenic flies bearing the Mtnv $v^{k}$ LTR construction. Each lane contains $5 \mu \mathrm{g}$ of poly $(\mathrm{A})^{+}$adult RNA isolated from the strains indicated. The $s u(s)^{-}$ $r^{500} v^{36 f}$ strain is the transformation host strain in a $s u(s)^{-}$mutant background. Mtnv ${ }^{k}$ LTR-1 and Mtnv ${ }^{k}$ LTR-2 are two independent transgenic lines. The Northern blot was simultaneously hybridized with radiolabeled $v$ and $r p 49$ antisense riboprobes (see Materials and Methods). The positions of the $v$ transcripts which retain the 412 LTR $(1.9 \mathrm{~kb})$ and those which have the 412 LTR sequences removed by splicing $(1.4 \mathrm{~kb})$ are indicated. The internal control $r p 49$ transcripts are also indicated. 
do not affect the apparent efficiency at which the 412 LTR sequences are spliced from the RNA.

A trivial explanation for the observed increases in the accumulation of Mtnv ${ }^{k} L T R$ transcripts is that $s u(s)$ mutations increase the frequency of transcription initiation at the Mtn promoter. To address this possibility, this and other Northern blots were probed with Mtn sequences. Mutations at the $s u(s)$ gene did not affect the levels of the endogenous $M t n$ transcripts (data not shown), indicating that $s u(s)$ is not a regulator of $M t n$ transcription and that the increase in the accumulation of the Mtnv ${ }^{\mathrm{k}} \mathrm{LTR}$ transcripts in a su(s) mutant background is most likely not occurring through increased transcription initiation at the Mtn promoter.

Analysis of a construction with a consensus $5^{\prime}$ splice site sequence. The finding that $s u(s)$ mutations affect the Mtnv ${ }^{k}$ LTR construction by increasing the accumulation of both fully spliced transcripts and transcripts which retain the 412 LTR sequences might be explained if $s u(s)$ mutations increase the stability of the LTR-containing RNA. For example, if the $s u(s)$ protein participates directly in the degradation of mutant $v$ transcripts, the stability of these transcripts would be increased in $s u(s)$ mutants. Alternatively, the stability of the mutant $v$ transcripts might be coupled with pre-mRNA splicing, such that the formation of more stable splicing complexes on cryptic splice sites in $s u(s)$ mutants protects the transcripts from degradation. To determine whether the stability of the RNA and the effect of $s u(s)$ are related to splicing, we constructed several derivatives of Mtnv $^{\mathrm{k}}$ LTR with alterations in the $\mathbf{4 1 2}$ cryptic splice sites and used them to generate transformants. In one construction, the region of cryptic $4125^{\prime}$ splice donor sites I and II was converted to a consensus $5^{\prime}$ splice site sequence CAG/ GTAAGT (26) and the highly conserved GT dinucleotides of the other previously identified 412 cryptic $5^{\prime}$ splice sites in the Mtnv ${ }^{k} L T R$ construction were changed to CA dinucleotides (Fig. 1B, Mtnv ${ }^{k}$ LTR5' $^{\prime}$ con). This construction is otherwise identical to $M t n v^{k} L T R$. If $s u(s)$ mutations produce the same effect on the accumulation of transcripts with the consensus $5^{\prime}$ splice site sequence as on the accumulation of transcripts with the cryptic $4125^{\prime}$ splice sites (Mtnv ${ }^{k}$ LTR), this would suggest that suppression does not directly involve recognition of the cryptic $4125^{\prime}$ splice sites. However, if the $s u(s)$ protein functions to prevent splicing complexes from forming on poor $5^{\prime}$ splice sites but does not affect recognition of good sites, then the effect of $s u(s)$ mutations on transcripts with the consensus $5^{\prime}$ splice site would be expected to be less dramatic. A Northern blot with RNA isolated from transgenic flies bearing the Mtnv ${ }^{k} L T R 5$ 'con construction is shown in Fig. 3. Strikingly, introduction of the consensus 5' splice site sequence produced the same effect as mutations at the $s u(s)$ gene: the levels of both the fully spliced transcripts and transcripts which retain the 412 LTR sequences were increased (Fig. 3; compare lanes 3 and 5 with lane 8). The observed expression pattern, including the ratio of the fully spliced to LTR-containing transcripts, was similar to that observed with the Mtnv ${ }^{k}$ LTR construction in a su(s) mutant background (Fig. 3; compare lanes 3 to 6 with lane 8). Furthermore, the effect of $s u(s)$ mutations on the accumulation of the transcripts produced from Mtnv ${ }^{k} \mathbf{L T R 5}$ con was virtually eliminated (Fig. 3; compare lane 3 with lane 4 and lane 5 with lane 6 ). Taken together, these results suggest that $s u(s)$ mutations and the $5^{\prime}$ splice site consensus sequence affect the accumulation of the mutant $v$ transcripts through the same process. Replacing the $5^{\prime}$ cryptic donor sites with a consensus sequence, a change which should enhance complex assembly, leads to higher RNA levels without appar-

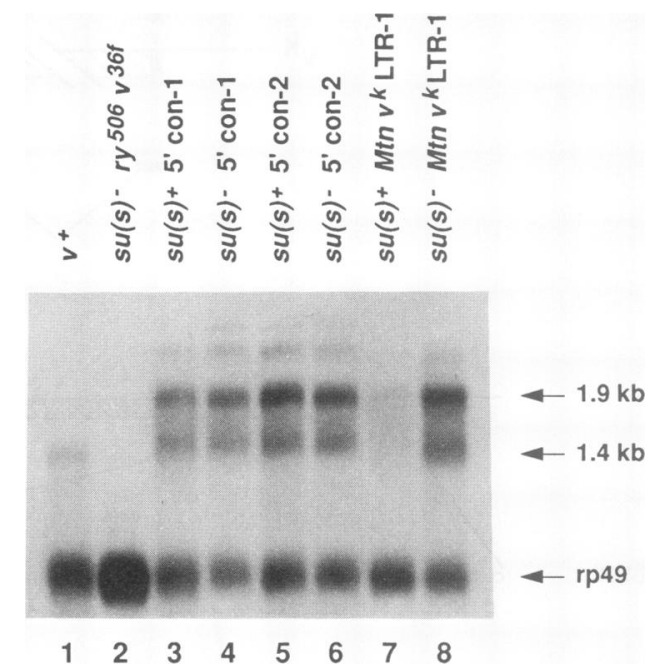

FIG. 3. Northern blot analysis of RNA isolated from transgenic flies bearing the Mtnvk ${ }^{k}$ TR5' con construction. Each lane contains 2 $\mu \mathrm{g}$ of $\operatorname{poly}(\mathrm{A})^{+}$adult RNA isolated from the strains indicated. Two independent $M$ Mnv $^{\mathrm{k}}{ }^{\mathrm{LTR}}$ ' con transgenic lines are indicated as 5 'con-1 and 5'con-2. The blot was simultaneously hybridized with $v$ and $r p 49$ antisense riboprobes. The positions of the $v$ transcripts which retain the 412 LTR sequences $(1.9 \mathrm{~kb})$ and those which have the LTR sequences removed by splicing $(1.4 \mathrm{~kb})$ are indicated. The internal control $r p 49$ transcripts are also indicated.

ently affecting the efficiency of splicing out the 412 intron. In mutants lacking the $s u(s)$ protein, the same effect could be a consequence of increased complex assembly on the cryptic donor sites.

Analysis of constructions with mutations in the previously identified 412 cryptic $5^{\prime}$ and $3^{\prime}$ splice sites. If assembly of 412 LTR sequences into splicing complexes stabilizes the $v$ mutant transcripts, then elimination of these cryptic splice sites would be predicted to lead to reduced RNA accumulation. Furthermore, if all of the potential splice sites in the 412 LTR sequences were completely eliminated, then according to our model, $s u(s)$ mutations would not be expected to affect accumulation of the transcripts produced. To test these predictions, two constructions, bearing mutations designed to eliminate the cryptic $4125^{\prime}$ and $3^{\prime}$ splice sites, were made. In the first of these (Mtnv ${ }^{k} L T R \Delta 5^{\prime}$ SS; Fig. 1B), the highly conserved GT dinucleotides of $\mathbf{4 1 2}$ donor sites II, III, and IV in the Mtnv ${ }^{k} L T R$ construction were changed to CA dinucleotides. Also, a G-to-C mutation was introduced into the GT dinucleotide of 412 donor I. The second construction, Mtnv ${ }^{k} L T R \Delta 5^{\prime}, 3^{\prime}$ SS, has these same mutations of the $4125^{\prime}$ splice sites but also has a G-to-C mutation of the highly conserved AG dinucleotide of the $4123^{\prime}$ splice site (Fig. 1B). Unexpectedly, when poly(A) ${ }^{+}$RNA isolated from transgenic flies bearing the Mtnv ${ }^{k}$ LTR $\Delta 5^{\prime} S S$ construction was examined by Northern blot analysis, $v$-specific transcripts corresponding in size to those which retain the 412 LTR sequences and those which have the 412 LTR sequences removed by splicing were observed (Fig. 4A). In fact, the pattern of transcripts observed from the $M^{\prime \prime n v} v^{k} L T R \Delta 5^{\prime} S S$ construction and the effect of $s u(s)$ mutations on transcript accumulation were very similar to those for the original Mtnv ${ }^{k}$ LTR construction. To determine how the apparently spliced transcripts were generated, firststrand $v$-specific cDNA products of the Mtnv ${ }^{\mathrm{k}} \mathrm{LTR} \Delta 5^{\prime} \mathrm{SS}$ construction were PCR amplified and sequenced. This anal- 
A.

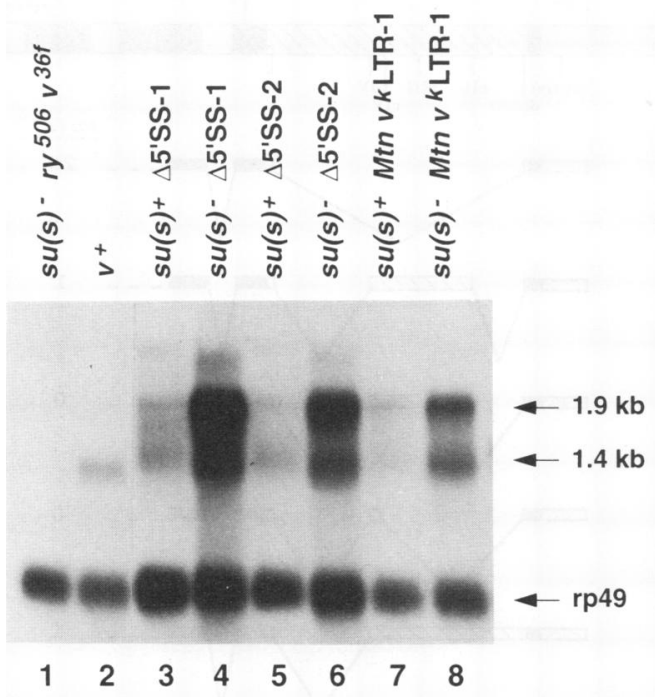

B.

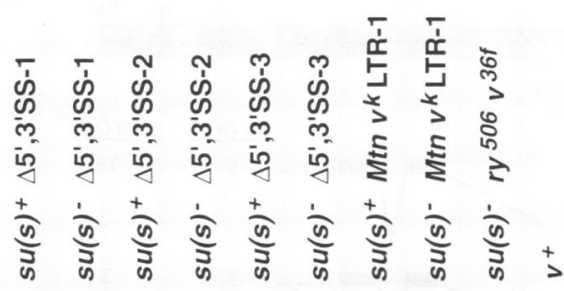

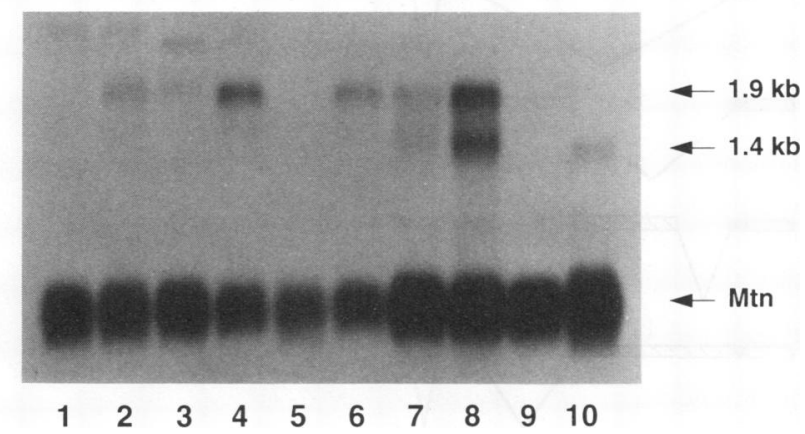

FIG. 4. Northern blot analysis of transgenic flies bearing the Mtnv ${ }^{k} L T R \Delta 5^{\prime} S S(A)$ and Mtnv $v^{k} L T R \Delta 5^{\prime}, 3^{\prime} S S$ constructions (B). Each lane contains $2 \mu \mathrm{g}$ of poly $(\mathrm{A})^{+}$adult RNA isolated from the strains indicated. $\Delta 5^{\prime}$ SS-1 and $\Delta 5^{\prime} \mathrm{SS}-2$ represent two independent Mtnv ${ }^{k} L T R \Delta 5^{\prime} S S$ transgenic lines; $\Delta 5^{\prime}, 3^{\prime}$ SS-1, -2 , and -3 represent three independent Mtnv $v^{k}$ LTR $\Delta 5^{\prime}, 3^{\prime}$ 'SS transgenic lines. The blots were simultaneously hybridized with $v$ and $r p 49$ (A) or $v$ and $M t n$ (B) antisense riboprobes. The positions of the $v$ transcripts which retain the 412 LTR sequences $(1.9 \mathrm{~kb})$ and those which have the LTR sequences removed by splicing $(1.4 \mathrm{~kb})$ are indicated. The positions of the internal control $r p 49$ and Mtn transcripts are also indicated.

ysis revealed that splicing occurred from a cryptic 5' splice site (CAA/GTGAAT) located within the Mtn leader region to the previously described $4123^{\prime}$ splice site near the end of the 412 LTR (Fig. 1B). This $5^{\prime}$ splice site is located $29 \mathrm{nt}$ downstream of the transcription start site and is not a site that is normally used in processing Mtn transcripts (14). Furthermore, this site is present in all of the Mtnv ${ }^{\mathrm{k}} \mathbf{L T R}$ derivatives, and it is also used in splicing transcripts produced from Mtnvk LTR but apparently not from Mtnv ${ }^{k}$ LTR5' con (see below). Thus, while the changes introduced into this construction did not prevent the 412 LTR from being recognized as an intron by the splicing machinery, this analysis demonstrated that the effect of $s u(s)$ mutations is not specific for the cryptic $4125^{\prime}$ splice sites.

Poly $(\mathrm{A})^{+}$RNA was also analyzed from transgenic flies bearing the Mtnvk LTR $\Delta 5^{\prime}, 3^{\prime}$ SS construction, which has mutations in both the $4125^{\prime}$ and $3^{\prime}$ splice sites but retains an intact cryptic Mtn $5^{\prime}$ splice site. A Northern blot of this RNA (Fig. 4B) showed that only the LTR-containing 1.9-kb transcript accumulates; thus, splicing of the 412 LTR sequences was largely abolished. The overall level of RNA accumulation is significantly reduced relative to $M \operatorname{Mnv}^{k} \mathbf{L T R}$ in both $s u(s)$ wild-type and mutant backgrounds. This finding supports the notion that splicing complex assembly stabilizes the LTR-containing transcripts. The level of accumulation of these transcripts is higher in a su(s) mutant background (Fig. $4 \mathrm{~B}$, lanes 2,4 , and 6$)$. If the effect of $s u(s)$ mutations involves splicing complex assembly on cryptic sites, perhaps an effect is observed on Mtnv $v^{k} L T R \Delta 5^{\prime}, 3^{\prime}$ SS transcripts because all of the cryptic splice sites were not eliminated by the alterations in the $\mathbf{4 1 2}$ donor and acceptor sites. As described above, the cryptic Mtn donor site is present in this construction, and analysis of PCR-amplified cDNA clones isolated from transformants bearing the Mtnv ${ }^{k} L T R \Delta 5^{\prime}, 3^{\prime}$ SS construction revealed that a low level of splicing of the 412 LTR sequences does occur. Of three cDNA clones obtained that had the 412 LTR sequences removed by splicing, two were spliced at the Mtn cryptic 5' splice site and at a $3^{\prime}$ TG dinucleotide located 4 to 5 bp upstream of the mutated $4123^{\prime}$ splice site, and the third was spliced at the Mtn $5^{\prime}$ splice site and at a $3^{\prime}$ AT dinucleotide located 5 to $6 \mathrm{bp}$ downstream of the mutated $4123^{\prime}$ splice site (Fig. 1B). Thus, even in the absence of the $4125^{\prime}$ and $3^{\prime}$ splice sites, a low level of splicing of the 412 sequences does occur. Furthermore, as will be described below, other cryptic sites within the LTR can mediate splicing complex assembly.

Splicing patterns of transcripts produced by the Mtnv' LTR and Mtnv'LTR5'con constructions. The observation that transcripts produced by the construction with mutations of the $4125^{\prime}$ splice sites (Mtnvk ${ }^{k}$ TR $\Delta 5^{\prime} \mathrm{SS}$ ) are spliced at a cryptic 5' splice site located within the Mtn leader sequences suggested that this splice site may also be used to splice transcripts produced by the Mtnvk LTR and Mtnv ${ }^{k} L T R 5^{\prime}$ con constructions. To determine the splicing patterns of these transcripts, several PCR-amplified CDNA clones of each of these constructions were analyzed. In the majority of the Mtnv $^{k}$ LTR cDNA clones, the 412 LTR sequences were spliced from either the Mtn cryptic 5' splice site or 412 donor site II to the previously described $4123^{\prime}$ splice site near the end of the 412 LTR (Fig. 5). However, several cDNA clones, obtained from both $s u(s)$ wild-type and $s u(s)$ mutant RNAs, were spliced at previously unidentified cryptic splice sites located internally within the 412 LTR sequences. These additional splice sites, including three acceptor sites and one donor site, are shown in Fig. 5.

Most of the Mtnv ${ }^{k} L T R 5^{\prime}$ con cDNA clones were spliced from the consensus $5^{\prime}$ splice site to the $4123^{\prime}$ splice site located near the end of the 412 LTR (Fig. 6). In fact, in the presence of the $5^{\prime}$ consensus splice site, none of the cDNA clones isolated were spliced at the cryptic Mtn donor site. 
A.

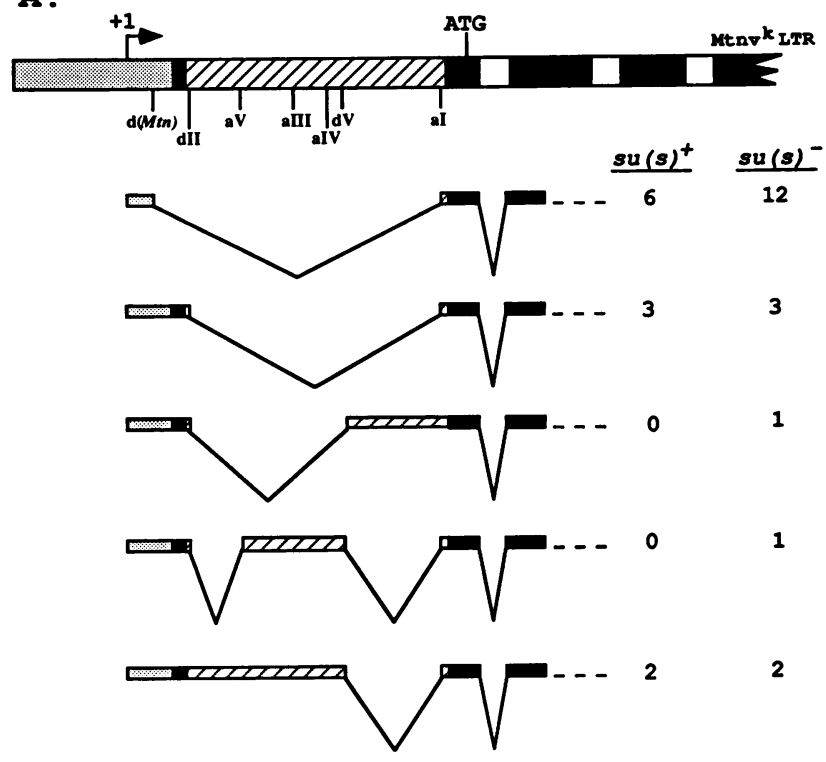

B.

\begin{tabular}{|c|c|c|c|}
\hline $\begin{array}{l}\text { donor } \\
\text { sites }\end{array}$ & sequence & $\begin{array}{l}\text { acceptor } \\
\text { sites } \\
\end{array}$ & sequence \\
\hline$d(M t n)$ & CAA/GTGAAT & aI & TTGTTCTTATTATTGCATAG/G \\
\hline dII & CGT/GTAATG & aIv & TGTATATCTTATATATCTAG/G \\
\hline dv & TAT/GTACGT & av & TTCTTTTGATTTTAGAGTAG/G \\
\hline
\end{tabular}

FIG. 5. Splicing patterns of Mtnv ${ }^{k}$ LTR cDNAs. (A) Schematic diagram of the Mtnvk LTR construction. Positions of the donor (d) and acceptor (a) sites used to splice the 412 LTR sequences are indicated. The 412 LTR sequences are represented by the hatched box, $v$ exons are represented by shaded boxes, and $v$ introns are represented by open boxes. Mtn gene promoter and leader sequences are represented by the stippled box. The cryptic donor splice site contained within the untranslated Mtn leader sequences is labeled $\mathrm{d}(M t n)$. Below the diagram, the splicing patterns of PCRamplified cDNA clones are shown, with the numbers of clones of each type isolated from $s u(s)^{+}$and $s u(s)^{-}$backgrounds shown at the right. In each case, the sequence of a cDNA fragment extending from a ClaI site in $v$ exon 3 to the $5^{\prime}$ Mtn leader region was determined. The first and second authentic $v$ introns were properly spliced from all of these cDNAs. (B) Sequences at the junctions of the cryptic donor and acceptor splice sites.

This finding indicates that the $5^{\prime}$ consensus is, in fact, recognized more efficiently than the cryptic $5^{\prime}$ splice sites in vivo. However, as with $M_{n v^{k}}{ }^{2} T R$, several cDNA clones that were spliced at the cryptic splice sites located internally within the 412 LTR were also obtained (Fig. 6). Interestingly, some of these internal cryptic 412 splice sites, particularly acceptor II and donor V (Fig. 6), are better matches to the consensus splice site sequences than the previously described splice sites located near the ends of the 412 LTR (Fig. 1). It might be argued that since these cDNA clones were isolated by PCR amplification, splicing at these internal sites could occur very infrequently and represent a normal degree of sloppiness in the splicing process. However, our analysis of these same cDNAs with regard to splicing of the first authentic $v$ intron has not detected a single instance of splicing at unexpected sites. In addition, S1 nuclease protection analysis indicated that transcripts spliced at these sites within the 412 LTR represent a significant fraction of
A.

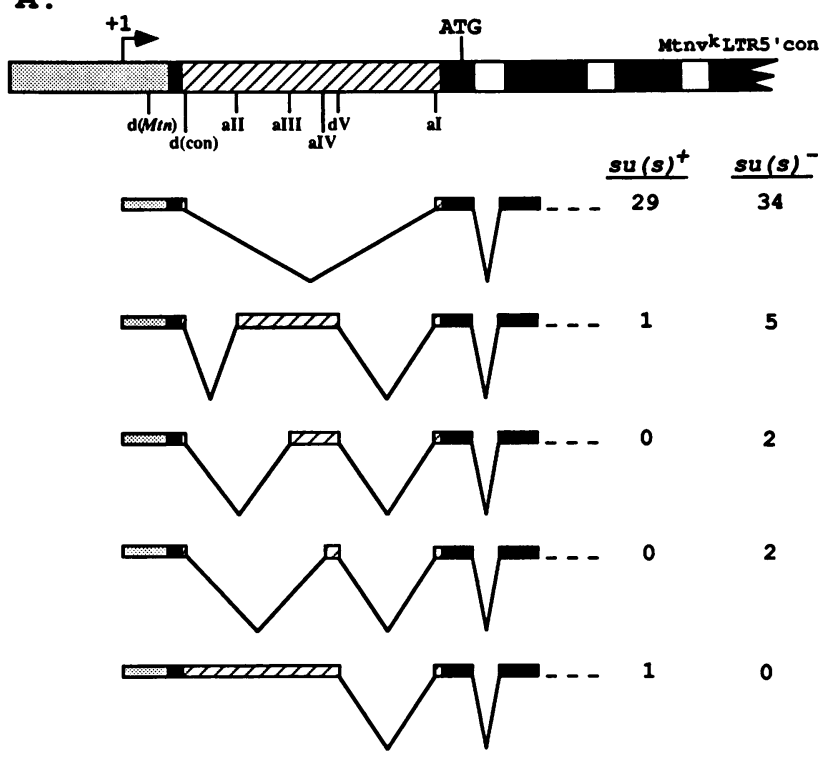

B.

\begin{tabular}{|c|c|c|c|}
\hline $\begin{array}{l}\text { donor } \\
\text { sites } \\
d(\operatorname{con})\end{array}$ & $\frac{\text { sequence }}{\text { CAG/GTAAGT }}$ & $\begin{array}{c}\text { acceptor } \\
\text { sites }\end{array}$ & $\frac{\text { sequence }}{\text { TTGTTCTTATTATTGCATAG/G }}$ \\
\hline \multirow[t]{3}{*}{$d v$} & TAT/GTACGT & aII & ATTTCTTCTTTTGATTTTAG/A \\
\hline & & aIII & TTTAATATATTTGGACTTAG/G \\
\hline & & arv & TGTATATCTTATATATCTAG/ \\
\hline
\end{tabular}

FIG. 6. Splicing patterns of the Mtnv ${ }^{k} L T R 5$ 'con cDNAs. (A) Schematic diagram of the Mtnv $v^{k}$ LTR5' con construction. Positions of the donor (d) and acceptor (a) sites used to splice the 412 LTR sequences are indicated. The symbols are as described in the legend to Fig. 5. In the Mtnvk LTR5'con construction, the region including 412 splice site donors I and II (see Fig. 1) was converted to a consensus $5^{\prime}$ splice site [d(con)]. Below the diagram, the splicing patterns of PCR-amplified CDNA clones are shown, with the numbers of clones of each type isolated from $s u(s)^{+}$and $s u(s)^{-}$backgrounds shown at the right. As in Fig. 5, the sequence of a cDNA fragment extending from a $\mathrm{Cla}$ I site in $v$ exon 3 to the $5^{\prime} \mathrm{Mtn}$ leader region was determined. The first and second authentic $v$ introns were properly spliced from all of these cDNAs. (B) Sequences at the junctions of the cryptic donor and acceptor splice sites. The conserved AG of aII is located only $5 \mathrm{nt}$ upstream of the splice site of aV (see Fig. 5).

the total population of mutant $v$ transcripts (data not shown). This type of analysis also indicated that $s u(s)$ mutations do not appreciably affect the proportion of transcripts spliced at these alternate sites as might be inferred from the data in Fig. 5 and 6 (data not shown). Perhaps these alternatively spliced transcripts produce the smearing observed on Northern blots between the 1.9-kb LTR-containing and 1.4-kb fully spliced $v$ transcripts (for example, Fig. 2 and 3).

\section{DISCUSSION}

In an attempt to define a function for the $s u(s)$ protein, we have examined the effect of $s u(s)$ mutations on expression of $s u(s)$-suppressible $v$ alleles. We previously showed that the three suppressible $v$ alleles have identical insertions of a 412 retrotransposon in the first $v$ exon and that the 412 sequences are removed by splicing. In this report, we have analyzed the 
effect of $s u(s)$ mutations on $v^{k}$ derivatives which were introduced into the germ line of $D$. melanogaster by P-element-mediated transformation. Expression of these constructions was driven by the stronger $M t n$ promoter. A construction with a single 480-bp 412 LTR at the insertion site of the 7.5-kb 412 element in the $v^{s}$ alleles (Mtnv ${ }^{k}$ LTR) produced two classes of polyadenylated transcripts, ones that retain 412 LTR sequences but have undergone normal splicing of $v$ introns and ones that have had the 412 LTR sequences as well as the $v$ introns spliced from the premRNA. Mutations at the $s u(s)$ gene result in increases in the accumulation of both classes of transcripts. Similar observations were made in the analysis of an allele of the gene, $y^{76 d 28}$, that is also suppressed by $s u(s)$ mutations (6). The $y^{76 d 28}$ allele has a P-element insertion in the $5^{\prime}$ transcribed leader region of the $y$ gene. As with the 412 insertion at $v$, the P-element sequences are inefficiently spliced from the premRNA at cryptic splice sites located near the ends of the transposable element. Mutations at $s u(s)$ result in increased accumulation of both P-element-containing transcripts and transcripts from which the $\mathrm{P}$ element has been removed by splicing (6). Thus, both of the examples of $s u(s)$-suppressible alleles that have been examined at the molecular level involve splicing of transposable element sequences at sites that are not normally utilized as splice sites in the processing of pre-mRNAs. Furthermore, $s u(s)$ mutations similarly affect the levels of transposon-containing and fully spliced transcripts. In theory, the increase in transposon-containing transcripts observed in $s u(s)$ mutants could be due to an effect on transcription or on transcript stability. However, our finding that replacing a cryptic $5^{\prime}$ donor with a consensus site eliminates the effect of $s u(s)$ mutations provides compelling evidence that $s u(s)$ mutations act posttranscriptionally, i.e., by affecting RNA stability, and that stability is somehow dependent on splicing complex assembly.

Support for the notion that LTR-containing transcripts are unstable and that stability is enhanced by assembly of the LTR into splicing complexes was obtained from analysis of the LTR derivatives with splice site alterations. The fact that the addition of a consensus splice donor site resulted in increased levels of LTR-containing transcripts indicates that the stability of these transcripts can be increased by more stable complex assembly at the $5^{\prime}$ donor site. Perhaps this, in turn, leads to a higher level of mRNA accumulation, since retention of the transcript in splicing complexes would prevent degradation and allow more time for splicing to occur. Furthermore, transcripts produced from the construction with $5^{\prime}$ and $3^{\prime}$ splice site alterations that were designed to prevent splicing complex assembly (Mtnv ${ }^{\mathbf{k}}$ LTR $\Delta 5^{\prime}, 3^{\prime}$ SS) accumulated reduced levels of LTR-containing transcripts. This result also suggests that splicing complex assembly stabilizes these transcripts.

The $s u(s)$ protein could either play a direct role in controlling RNA stability, for example, if it is part of the RNA degradation machinery, or function in splicing complex assembly and therefore affect RNA stability indirectly. The results obtained from analysis of the LTR derivative in which a $5^{\prime}$ consensus site was substituted for one of the cryptic 5' splice sites (construction Mtnv $^{\mathrm{k}}$ LTR5'con) are consistent with the hypothesis that $s u(s)$ mutations affect the stability of splicing complexes. The overall levels and relative amounts of LTR-containing and fully spliced transcripts produced by the Mtnv ${ }^{k}$ TR5 $^{\prime}$ con were similar to those observed from the construction with the cryptic 412 splice sites (Mtnvk ${ }^{k}$ TR) in a $s u(s)$ mutant background. Thus, the addition of the $5^{\prime}$ splice site consensus sequence mimics the effect of $s u(s)$ mutations. Moreover, $s u(s)$ mutations did not appreciably affect the accumulation of transcripts produced by the construction with the $5^{\prime}$ consensus splice site. Since the effect of the $5^{\prime}$ splice site consensus sequence almost certainly occurs at the level of pre-mRNA splicing, it seems probable that mutations at the $s u(s)$ gene also affect an aspect of splicing.

Interestingly, neither $s u(s)$ mutations nor the addition of the consensus $5^{\prime}$ splice site sequence appreciably affects the apparent efficiency of splicing of the 412 sequences, as judged by the relative ratios of fully spliced transcripts to transcripts that retain the 412 LTR sequences (Fig. 2 and 3). A possible explanation for this is that $s u(s)$ mutations and the $5^{\prime}$ splice site consensus sequence increase the stability of transcripts which retain the 412 sequences without affecting the actual rate of splicing of the 412 sequences. It is possible, for example, that the presence of the 412 sequences destabilizes the mutant $v$ transcripts but that the formation of splicing complexes on the 412 sequences protects them from degradation. The effect of $s u(s)$ mutations and the consensus $5^{\prime}$ splice site sequence might be to increase the efficiency of an early recognition step without affecting the rate-limiting step in the splicing of the 412 sequences.

On the surface, the results obtained from the construction with mutations of the previously identified $4125^{\prime}$ and $3^{\prime}$ cryptic splice sites $\left(\mathrm{Mtnv}^{\mathrm{k}} \mathrm{LTR} \Delta 5^{\prime}, 3^{\prime} \mathrm{SS}\right)$ appear to argue against the possibility that the $s u(s)$ protein functions at the level of pre-mRNA splicing. By Northern blot analysis, splicing of the 412 sequences from transcripts produced by this construction appears to be largely prevented, yet $s u(s)$ mutations still affect the accumulation of these transcripts. The interpretation of these results is, however, complicated because the alterations that we introduced probably did not completely eliminate splicing complex assembly. First, since the cryptic $5^{\prime}$ splice site located within the Mtn leader sequences is intact in the $M \operatorname{Mnv}^{\mathrm{k}} \mathrm{LTR} \Delta 5^{\prime}, 3^{\prime} \mathrm{SS}$ construction, it is possible that even in the absence of the AG dinucleotide at the $3^{\prime}$ splice site, splicing complexes can assemble on the Mtn cryptic donor site. This possibility is supported by the finding that a low level of splicing of the 412 sequences from the Mtnv ${ }^{k} L T R \Delta 5^{\prime}, 3^{\prime} S S$ transcripts occurs from the cryptic Mtn $5^{\prime}$ splice site to non-AG $3^{\prime}$ splice sites located near the mutated $4123^{\prime}$ splice site. An increase in the stability of splicing complexes on this $5^{\prime}$ splice site could explain the effect of $s u(s)$ mutations on the accumulation of

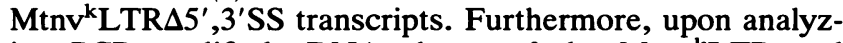
ing PCR-amplified cDNA clones of the Mtnvk ${ }^{k}$ TTR and Mtnv $^{\mathrm{k}}$ LTR5' $^{\prime}$ con constructions, we identified five additional $3^{\prime}$ splice sites and another $5^{\prime}$ splice site within the 412 LTR sequences (Fig. 5 and 6 ). In transcripts produced by the $\mathrm{Mtnv}^{\mathrm{k}} \mathrm{LTR} \Delta 5^{\prime}, 3^{\prime} \mathrm{SS}$ construction, particularly in a $s u(s) \mathrm{mu}-$ tant background, these sites may be recognized and splicing complexes could associate with the cryptic Mtn 5' splice site and one of the three internal $4123^{\prime}$ splice sites.

As stated above, it is also possible that the $s u(s)$ protein plays a direct role in degrading transcripts that are not assembled into splicing complexes. This hypothesis can readily explain why the ratio of LTR-containing (unspliced) to fully spliced transcripts is not affected in $s u(s)$ mutants. However, the results obtained with the Mtnv ${ }^{k} L T R \Delta 5^{\prime}, 3^{\prime} S S$ construction are not consistent with $s u(s)$ being directly involved in RNA degradation. If $s u(s)$ mutations inactivate a protein involved in targeting the LTR-containing transcripts for degradation, then in $s u(s)$ mutants, the overall level of transcript accumulation produced by the constructions with splice sites deleted should be the same as that produced by 


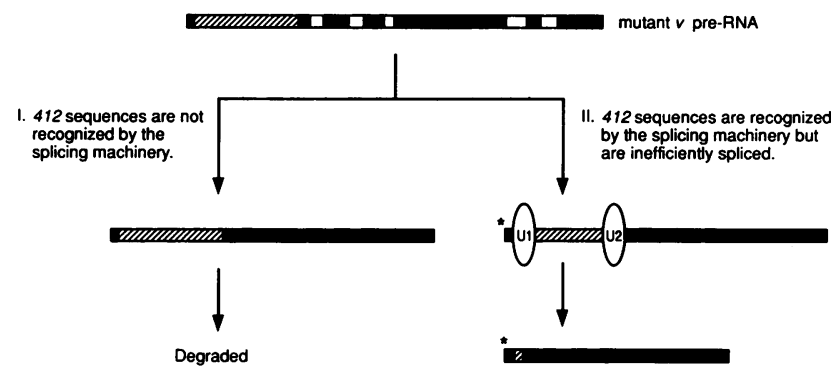

FIG. 7. Model for suppression of the mutant $v$ alleles. In the schematic diagram of the mutant $v$ pre-mRNA (top), the 412 LTR sequences are indicated by the hatched box, the $v$ exons are indicated by shaded boxes, and the $v$ introns are indicated by open boxes. Two possible fates (I and II) of the pre-mRNA are illustrated. In the first pathway (I), the $v$ introns are recognized and removed by the splicing machinery, but the 412 sequences are not recognized as intron sequences and are retained. The presence of the 412 sequences results in the rapid degradation of these transcripts. In the second pathway (II), the 412 sequences as well as the $v$ introns are recognized by the splicing machinery. The formation of splicing complexes on the 412 sequences stabilizes these transcripts, but the 412 sequences are spliced more slowly than the $v$ introns. The transcripts observed by Northern analysis are indicated with asterisks. These include transcripts that are complexed by the splicing machinery but still retain the 412 LTR sequences and those from which both the $v$ introns and the 412 sequences have been spliced. The effect of $s u(s)$ mutations is to increase the efficiency at which the 412 LTR sequences are recognized as intron sequences and hence to increase the proportion of pre-mRNA molecules which follow the second pathway (II).

the construction with the splice sites intact. In contrast, we observe that the level of transcripts with the mutated splice sites is significantly lower in both $s u(s)$ wild-type and mutant backgrounds. This finding indicates that the degradation of these transcripts is not eliminated by $s u(s)$ mutations. However, this interpretation makes the assumption that transcripts with the splice sites destroyed are degraded by the same pathway as those transcripts with splice sites intact, and this might not be the case. Another argument against a role for the $s u(s)$ protein in RNA degradation is that $s u(s)$ mutations are also known to enhance the phenotype of certain other mutant alleles (23). It is difficult to envision how the inactivation of a degradation pathway could lead to reduced production of a particular gene product. Conversely, altering splicing complex assembly would generally be expected to negatively effect gene expression. A third argument against a direct role for $s u(s)$ in RNA degradation is that the degradation activity would have to be specific, since only the levels of certain transcripts are affected; this possibility seems unlikely. Thus, we favor the hypothesis that $s u(s)$ encodes a protein that functions in the splicing pathway. However, biochemical analysis, currently under way, will be needed to determine unambiguously the function of the $s u(s)$ protein.

A model to explain the effect of $s u(s)$ mutations on the expression of the Mtn: $v^{k}$ derivatives is shown in Fig. 7. According to this model, in a su(s) wild-type background, the 412 sequences in the mutant $v$ transcripts would rarely be recognized by the splicing machinery and most of the transcripts would be rapidly degraded (pathway I). When the 412 sequences are recognized as an intron, the assembly of the 412 sequences into a splicing complex stabilizes the transcripts but splicing of the 412 sequences is inefficient (pathway II). In a $s u(s)$ mutant background, the proportion of $v$ transcripts that enter the splicing pool (pathway II) increases, but these transcripts are still inefficiently spliced. An important feature of this model is the assumption that the presence of the 412 sequences in the $v$ pre-mRNA results in the degradation of these transcripts. Potentially, this degradation could occur in either the nucleus or the cytoplasm. If the 412-containing transcripts are degraded in the nucleus, the formation of splicing complexes on the transposon sequences might protect the transcripts from nuclear RNases. If, on the other hand, degradation occurs in the cytoplasm, the retention of the 412 LTR-containing transcripts in the nucleus through their association with the splicing machinery might explain the increase in their stability. In either case, the increased assembly of the 412 sequences into splicing complexes in a su(s) mutant background could stabilize the transcripts. This model is consistent with the previously proposed role of the $s u(s)$ protein in destabilizing splicing complexes on the poor $4125^{\prime}$ splice sites (3). In this regard, the $s u(s)$ protein might function as a proofreading factor for $5^{\prime}$ splice site recognition. However, it is also possible that $s u(s)$ mutations affect an aspect of intron recognition that is not related to the poor $4125^{\prime}$ splice sites.

The process of how actual splice sites are distinguished from other sequences within a pre-mRNA that resemble splice sites but are not recognized as such is poorly understood (for a review, see reference 7). Recently, several factors that influence $5^{\prime}$ splice site selection have been identified $(5,10,15)$. The results presented in this report suggest that the $s u(s)$ protein may also play a role in this process.

\section{ACKNOWLEDGMENTS}

We thank G. Maroni, P. Pukkila, and R. Kole for critically reading the manuscript, M. Peifer and members of the Searles laboratory for helpful discussions, M. Khashab for technical assistance, and S. Whitfield for the artwork.

This work was supported by grants DMB-9004708 and MCB9205425 from the National Science Foundation.

\section{REFERENCES}

1. Bingham, P. M., R. Levis, and G. M. Rubin. 1981. Cloning of DNA sequences from the white locus of $D$. melanogaster by a novel and general method. Cell 25:693-704.

2. Feinberg, A. P., and B. Vogelstein. 1983. A technique for radiolabeling DNA restriction endonuclease fragments to high specific activity. Anal. Biochem. 132:6-13.

3. Fridell, R. A., A.-M. Pret, and L. L. Searles. 1990. A retrotransposon 412 insertion within an exon of the Drosophila melanogaster vermilion gene is spliced from the precursor RNA. Genes Dev. 4:559-565.

4. Fridell, Y.-W. C., and L. L. Searles. 1992. In vivo transcriptional analysis of the TATA-less promoter of the Drosophila melanogaster vermilion gene. Mol. Cell. Biol. 12:4571-4577.

5. Ge, H., P. Zuo, and J. L. Manley. 1991. Primary structure of the human splicing factor ASF reveals similarities with Drosophila regulators. Cell 66:373-382.

6. Geyer, P. K., A. J. Chien, V. G. Corces, and M. M. Green. 1991. Mutations in the su(s) gene affect RNA processing in Drosophila melanogaster. Proc. Natl. Acad. Sci. USA 88:7116-7120.

7. Green, M. R. 1991. Biochemical mechanisms of constitutive and regulated pre-mRNA splicing. Annu. Rev. Cell Biol. 7:559-599.

8. Higuchi, R. 1990. Recombinant PCR, p. 177-183. In M. A. Innis, D. H. Gelfand, J. J. Sninsky, and T. J. White (ed.), PCR protocols: a guide to methods and applications. Academic Press, Inc., San Diego, Calif.

9. Karess, R. E. 1985. P element mediated germ line transformation of Drosophila, p. 121-141. In D. M. Glover (ed.), DNA cloning: a practical approach, vol. 2. IRL Press, Oxford.

10. Krainer, A. R., A. Mayeda, D. Kozak, and G. Binns. 1991. 
Functional expression of cloned human splicing factor SF2: homology to RNA binding proteins, U1 70K, and Drosophila splicing regulators. Cell 66:383-394.

11. Kreitman, M., and L. F. Landweber. 1989. A strategy for producing single-stranded DNA in the polymerase chain reaction: a direct method for genomic sequencing. Gene Anal. Tech. 6:84-88.

12. Lindsley, D. L., and G. G. Zimm. 1992. The genome of Drosophila melanogaster. Academic Press, Inc., San Diego, Calif.

13. Maniatis, T., E. F. Fritsch, and J. Sambrook. 1982. Molecular cloning: a laboratory manual. Cold Spring Harbor Laboratory Press, Cold Spring Harbor, N.Y.

14. Maroni, G., E. Otto, and D. Lastowski-Perry. 1986. Molecular and cytogenetic characterization of a Metallothionein gene of Drosophila. Genetics 112:493-504.

15. Mayeda, A., and A. R. Krainer. 1992. Regulation of alternative pre-mRNA splicing by hnRNP Al and splicing factor SF2. Cell 68:365-375.

16. Melton, D. A., P. A. Krieg, M. R. Rebagliati, T. Maniatis, K. Zinn, and M. R. Green. 1984. Efficient in vitro synthesis of biologically active RNA and RNA hybridization probes from plasmids containing a bacteriophage SP6 promoter. Nucleic Acids Res. 12:7035-7056.

17. Mismer, D., and G. M. Rubin. 1987. Analysis of the promoter of the nina E. Opsin gene in Drosophila melanogaster. Genetics 116:565-578.

17a.Murray, M., and L. Searles. Unpublished data.

18. O'Connell, L. P., and M. Rosbash. 1984. Sequence, structure, and codon preference of the Drosophila ribosomal protein 49 gene. Nucleic Acids Res. 12:5495-5513.

19. O'Hare, K., R. Levis, and G. M. Rubin. 1983. Transcription of the white locus in Drosophila melanogaster. Proc. Natl. Acad. Sci. USA 80:6917-6921.

20. Phillips, J. P., and H. S. Forrest. 1980. Ommochromes and pteridines, p. 542-623. In M. Ashburner and T. R. F. Wright (ed.), The genetics and biology of Drosophila, vol. 2d. Academic Press, Inc., New York.

21. Pret, A.-M., and L. L. Searles. 1991. Splicing of retrotransposon insertions from transcripts of the Drosophila melanogaster vermilion gene in a revertant. Genetics 129:1137-1145.
22. Purugganan, M., and S. Wessler. 1992. The splicing of transposable elements and its role in intron evolution. Genetica 86:295303.

23. Rutledge, B. J., M. A. Mortin, E. Schwarz, D. Thierry-Mieg, and M. Meselson. 1988. Genetic interactions of modifier genes and modifiable alleles in Drosophila melanogaster. Genetics 119: 391-397.

24. Searles, L. L., R. S. Ruth, A. M. Pret, R. A. Fridell, and A. J. Ali. 1990. Structure and transcription of the Drosophila melanogaster vermilion gene and several mutant alleles. Mol. Cell. Biol. 10:1423-1431.

25. Searles, L. L., and R. A. Voelker. 1986. Molecular characterization of the Drosophila vermilion gene and its suppressible alleles. Proc. Natl. Acad. Sci. USA 83:404-408.

26. Shapiro, M., and P. Senapathy. 1987. RNA splice junctions of different classes of eukaryotes: sequence statistics and functional implications in gene expression. Nucleic Acids Res. 15:7155-7175.

27. Southern, E. M. 1975. Detection of specific sequences among DNA fragments separated by gel electrophoresis. J. Mol. Biol. 98:503-517.

28. Thomas, P. S. 1983. Hybridization of denatured RNA transferred or dotted to nitrocellulose paper. Methods Enzymol. 100:255-266.

29. Voelker, R. A., J. Gibson, J. P. Graves, J. F. Sterling, and M. Eisenberg. 1991. The Drosophila suppressor of sable gene encodes a 150-kilodalton polypeptide with regions similar to those of RNA-binding proteins. Mol. Cell. Biol. 11:894-905.

30. Voelker, R. A., J. Graves, W. Gibson, and M. Eisenberg. 1990. Mobile element insertions causing mutations in the Drosophila suppressor of sable locus occur in DNase I hypersensitive subregions of 5 '-transcribed nontranslated sequences. Genetics 126:1071-1082.

31. Walker, A. R., A. J. Howells, and R. G. Tearle. 1986. Cloning and characterization of the vermilion gene of Drosophila melanogaster. Mol. Gen. Genet. 202:102-107.

32. Zinn, K., D. Dimaio, and T. Maniatis. 1983. Identification of distinct regulatory regions adjacent to the human B-interferon gene. Cell 34:865-879. 